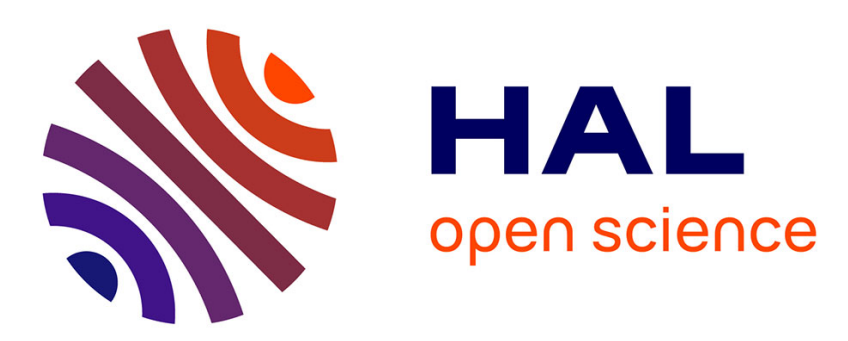

\title{
Planification de chaîne logistique sous incertitude: maintien de solution par satisfaction de contraintes dynamiques
}

\author{
Mariem Trojet, Fehmi H'Mida, Pierre Lopez, Patrick Esquirol
}

\section{To cite this version:}

Mariem Trojet, Fehmi H'Mida, Pierre Lopez, Patrick Esquirol. Planification de chaîne logistique sous incertitude: maintien de solution par satisfaction de contraintes dynamiques. Journal Européen des Systèmes Automatisés (JESA), 2016, 49 (6), pp.725-748. 10.3166/jesa.49.725-748 . hal-01398744

HAL Id: hal-01398744

https://hal.laas.fr/hal-01398744

Submitted on 17 Nov 2016

HAL is a multi-disciplinary open access archive for the deposit and dissemination of scientific research documents, whether they are published or not. The documents may come from teaching and research institutions in France or abroad, or from public or private research centers.
L'archive ouverte pluridisciplinaire HAL, est destinée au dépôt et à la diffusion de documents scientifiques de niveau recherche, publiés ou non, émanant des établissements d'enseignement et de recherche français ou étrangers, des laboratoires publics ou privés. 


\title{
Planification de chaîne logistique sous incertitude : maintien de solution par satisfaction de contraintes dynamiques
}

\author{
Mariem Trojet $^{1}$, Fehmi H'Mida ${ }^{1}$, Pierre Lopez ${ }^{2}$, Patrick Esquirol ${ }^{2}$ \\ 1. UR MSSDT / ENSIT / Université de Tunis \\ 5 avenue Taha Husein, BP 58, 1008, Tunis, Tunisie \\ mariemtrojet@hotmail.com,fehmi.hmida@enicarthage.rnu.tn \\ 2. LAAS-CNRS, Université de Toulouse, CNRS, INSA, Toulouse, France \\ pierre.lopez@laas.fr,patrick.esquirol@laas.fr
}

\begin{abstract}
RESUME. Ce travail porte sur la planification tactique d'une chaîne logistique dans un environnement incertain et perturbé. Dans le but de minimiser l'effet des perturbations dues à ces incertitudes, nous proposons une approche basée sur les problèmes de satisfaction de contraintes dynamiques. Il s'agit de planifier la production en recherchant le meilleur compromis entre les leviers décisionnels disponibles liés aux aspects capacité et coût de production en adoptant un processus dynamique qui permet d'actualiser les données à chaque étape de planification. Notre approche est évaluée par simulation de scénarios dans un contexte de données incertaines et de perturbations, influant notamment sur une variation de la demande et de la capacité. Les expérimentations mettent en balance une résolution statique et une résolution dynamique. La performance d'une solution de planification est renseignée par des mesures de la stabilité. Les résultats illustrent l'intérêt de l'approche basée sur la satisfaction de contraintes dynamiques.
\end{abstract}

\begin{abstract}
This work focuses on the supply chain tactical planning problem in an uncertain and turbulent environment. In order to minimize the effect of disturbances due to these uncertainties, we propose an approach based on dynamic constraint satisfaction problems. This is to plan production by searching the best compromise between available decision-making levers for capacity and production costs by adopting a dynamic process, which enables data update at each planning step. Our approach is evaluated by simulation under uncertain data. For this, we have developed various experiments related to the variation of customer demand and resource capacity. All the experiments are carried out by two different methods: a method based on a static CSP and a method based on a dynamic CSP. The performance of a planning solution is reported through stability measurement. Results of experiments confirm the performance of the method based on a dynamic CSP.
\end{abstract}

MOTS-CLES : chaîne logistique, planification, satisfaction de contraintes dynamiques, stabilité.

KEYWORDS: supply chain, planning, dynamic constraint satisfaction, stability. 


\section{Introduction}

La planification des chaînes logistiques consiste à prendre des décisions coordonnées tout au long de la chaîne et se décline traditionnellement selon trois niveaux hiérarchiques : le niveau stratégique, le niveau tactique et le niveau opérationnel (Tayur et al., 1999). Notre travail s'intéresse à la planification tactique d'une chaîne logistique. Il s'agit de planifier les volumes de production de chaque site pour chaque produit, les volumes de production sous-traités ainsi que le nombre d'heures supplémentaires utilisées à chaque période de l'horizon de planification, tout en respectant la capacité pour les différents sites de production. Chaque type de décision est associé à un coût que l'on cherche à minimiser.

Dans notre approche, nous souhaitons en premier lieu satisfaire toutes les contraintes posées au niveau tactique. Une autre préoccupation de notre travail est la prise en compte de l'incertitude. En effet, dans le contexte industriel actuel, certaines données mises à la disposition de la chaîne logistique telles que les temps de production ou de transport, les décisions prises par les partenaires, les capacités de production, ou les demandes clients peuvent être incertaines. (Galbraith, 1973) définit l'incertitude comme la différence entre la quantité d'information nécessaire pour effectuer une tâche et la quantité d'information dont on dispose déjà. Les entités de la chaîne sont ainsi soumises à des perturbations et aléas divers. En planification, il ne suffit plus d'avoir des plans qui optimisent les coûts. Les mesures portent aussi sur la quantification de la réorganisation induite par la modification d'un plan de production. Le maintien d'une solution est alors évalué par la comparaison entre : (1) les décisions engagées, correspondant au déroulement réel et prévu des activités de transformation du flux physique, et (2) les décisions du plan modifié, nécessitant la réorganisation et la mise à jour de ces mêmes activités suite à la modification d'une donnée.

Notre travail s'inscrit dans ce contexte de planification tactique établi dans un environnement dynamique. Plusieurs auteurs ont étudié les sources d'incertitudes présentes dans une chaîne logistique (Peidro et al., 2009) et les classifient selon trois catégories: l'incertitude sur la demande, l'incertitude sur le processus de fabrication comme les pannes machines, et l'incertitude sur l'approvisionnement liée par exemple aux retards de livraisons. Selon (Davis, 1993), l'incertitude sur la demande due à des prévisions inexactes est la plus importante.

Dans la littérature, la modélisation de l'incertitude en planification des chaînes logistiques est un champ de recherche auquel la communauté scientifique accorde une importance majeure. On trouve ainsi des modèles analytiques (optimisation robuste, programmation stochastique, théorie des jeux, programmation linéaire et paramétrique) (Al-Othman et al., 2008 ; Bihlmaier, 2009). Les incertitudes sont représentées par des distributions de probabilité tirées de l'historique des données. Ces données ne sont pas toujours fiables ou même disponibles, ce qui limite cette approche par détermination des distributions de probabilités. Dans ce contexte, les modèles d'intelligence artificielle (systèmes multi-agents, programmation linéaire floue, programmation multiobjectif floue, apprentissage par renforcement, algorithmes génétiques) (Peidro et al., 2010 ; 2009 ; Mitra et al., 2009), les modèles de simulation (simulation à événements discrets et systèmes dynamiques) (Suwanruji et al., 2006 ; Lim et al., 2006) et les modèles hybrides présentent des approches alternatives pour gérer les incertitudes en planification des chaînes logistiques.

Dans le cadre de ce travail et dans le but de maintien d'une solution face à des incertitudes, nous formulons notre modèle de planification d'une chaîne logistique comme un problème de 
satisfaction de contraintes dynamiques (DCSP, Dynamic Constraint Satisfaction Problem). En effet, un DCSP $(P)$ est une suite de CSP statiques où deux CSP successifs ne diffèrent que par un ajout ou un retrait de contraintes, variables, valeurs. Notre contribution par cette approche revient à utiliser la solution relative au problème initial pour la résolution du problème suivant sujet à des perturbations. L'approche est évaluée à l'aide d'indicateurs de performance en vue de quantifier les apports en termes de stabilité.

Pour cela, nous avons mené des expérimentations réalisées par deux méthodes de résolution différentes: une méthode de résolution statique et une méthode de résolution dynamique en exploitant les fonctionnalités d'un langage de programmation par contraintes. Ces expérimentations ont porté principalement sur les incertitudes liées à la variation de la demande clients.

L'article est organisé en quatre sections. La section 2 décrit les caractéristiques de la chaîne logistique considérée et définit le processus de planification dynamique. Dans la section 3, nous rappelons les principes de l'approche par satisfaction de contraintes. La section 4 est consacrée à la modélisation du processus de planification dynamique. La section 5 présente la méthodologie développée pour analyser la performance du modèle de planification dynamique d'une chaîne logistique. Nous présentons l'indicateur de stabilité employé pour le niveau planification. La section 6 porte sur l'analyse du modèle de planification dynamique et l'évaluation des deux approches de résolution CSP Statique/CSP Dynamique.

\section{Contexte de l'étude et analyse décisionnelle}

La chaîne logistique est un champ d'étude important qui a donné lieu à une littérature très abondante. Il n'y a pas une définition universelle de ce terme. Nous citons ci-dessous quelques définitions, issues de la littérature scientifique. Certains auteurs (Lee et Billington, 1993 ; RotaFrantz et al., 2001) assimilent une chaîne logistique à un réseau d'installations qui assure les fonctions d'approvisionnement en matières premières, de transformation de ces matières premières en composants puis en produits finis, et de distribution des produits finis vers le client.

Pour d'autres, (Génin, 2003 ; Stadtler et Kilger, 2000), la chaîne logistique est définie comme un réseau d'organisations ou de fonctions géographiquement dispersées sur plusieurs sites qui coopèrent, pour réduire les coûts et augmenter la vitesse des processus et activités entre les fournisseurs et les clients. Parmi les différentes définitions, celle de Lee et Bellington nous semble la plus adaptée à notre problématique : «Une chaîne logistique est un réseau d'installations qui assure les fonctions d'approvisionnement en matières premières, de transformation de ces matières premières en composants puis en produits finis, et de distribution des produits finis vers le client » (Lee et Bellington, 1993).

\subsection{Structure de la chaîne logistique}

La chaîne logistique considérée est limitée à trois types d'acteurs :

- une entreprise composée de plusieurs sites de production,

- un ensemble de clients pour l'entreprise,

- des sous-traitants pour chaque site de production. 
L'entreprise se compose de plusieurs sites de production réalisant différents types d'opérations de fabrication sur les produits. Plus précisément, on suppose que la réalisation d'un produit nécessite une succession d'opérations, chacune d'elles étant réalisée sur un site donné.

Un site est globalement défini par sa capacité de production. La capacité de production (i.e., de réalisation d'une opération) est le nombre de ressources (i.e., de machines) du site. Chaque site a la possibilité de recourir à la sous-traitance et/ou à l'utilisation d'heures supplémentaires. Dans le cas de la sous-traitance, le site ne fournit pas à son sous-traitant les composants nécessaires pour la fabrication des produits souhaités. La notion d'arriéré de production est prise en compte. Les arriérés sont assimilés à des quantités en rupture permettant de modéliser les retards dans la satisfaction des demandes.

\subsection{Processus et leviers décisionnels considérés}

Sur un horizon de planification discrétisé en périodes, il s'agit de planifier les volumes de produits à traiter, pour chaque produit et pour chaque site de production, tout en respectant les capacités des différents sites de production, qui sont définies sous la forme d'un nombre d'heures de travail disponibles. La capacité correspond à la définition d'un régime de fonctionnement nominal pour l'entreprise (i.e., un temps d'ouverture de 8 heures par jour et une seule équipe de travail).

La planification détermine aussi les volumes de production sous-traités et le nombre d'heures supplémentaires utilisées à chaque période de l'horizon de planification et pour chaque site de production. Les heures supplémentaires ne peuvent pas dépasser une limite maximale définie pour chaque site et pour chaque période. La capacité de sous-traitance est définie par un nombre de produits maximum par période. Nous introduisons un coût correspondant à chaque type de décision et c'est en termes de coût qu'une solution est considérée comme plus favorable qu'une autre : il s'agit de trouver une solution qui minimise le coût total des opérations.

\subsection{Processus de planification dynamique}

\subsubsection{Planification dynamique}

Classiquement, la planification de la production permet de définir des plans de production couvrant un horizon de planification $(H P)$ constitué de $T$ périodes. Ces plans sont obtenus en partant de certaines données généralement incertaines. Pour limiter l'influence de cette incertitude, nous adoptons dans le cadre de ce travail, un processus de planification dynamique qui nous permet de suivre et de contrôler la faisabilité des décisions prises.

La planification dynamique s'inscrit dans le cadre d'une procédure à horizon glissant permettant une actualisation périodique des données. En effet, les décisions prises sur un horizon de planification $H P$ de longueur $T$ sont remises en cause à intervalles de temps réguliers et une nouvelle planification est calculée sur un nouvel horizon de même longueur mais décalé de $\mathrm{Pa}$ périodes, $P a$ étant le pas de planification. Ainsi, lors de la première planification $(k=0)$, les plans sont calculés : le décideur reçoit le plan de demande prévu sur le premier horizon de planification $H P^{0}$ et détermine les différents plans sur l'ensemble des périodes appartenant à l'horizon $H P^{0}$. La mise à jour périodique des plans fait que toute nouvelle 
planification $(k)$ est réalisée sur un horizon de temps $H P^{k}=[\varphi, \varphi+1, \varphi+2, \ldots, \varphi+T-1]$ avec $\varphi=$ $k . P a+1$, et $k$ : numéro d'itération de la planification (figure 1) (Amrani-Zouggar, 2009).

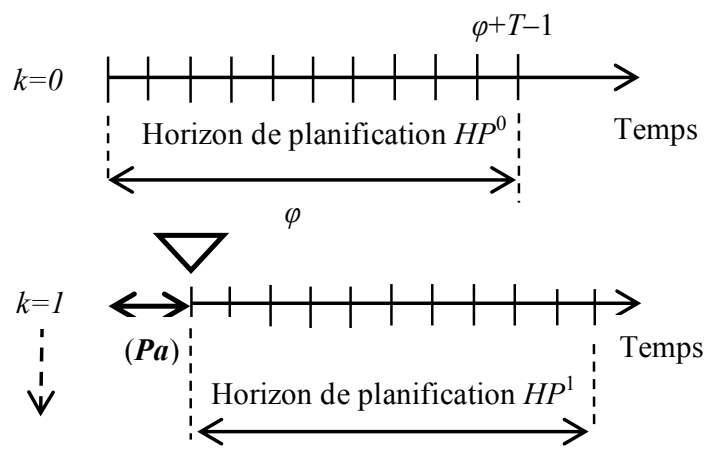

Figure 1. Processus de planification dynamique

\subsubsection{Procédure de planification}

Dans le cadre de ce travail, nous proposons une procédure itérative (figure 2) sur un horizon de planification $H P$ sur lequel les demandes sont supposées connues.

Un pas de planification $P a$ sépare deux itérations successives. La procédure débute par une initialisation des différentes données statiques et dynamiques du problème ainsi que la définition des stocks initiaux. La première planification $(k=0)$ est effectuée sur la base des données de l'horizon de planification correspondant $H P^{0}$ (demandes, coûts, capacités) commençant à la période $(T=1)$, avec $H P^{0}=\{1,2, \ldots, T\}$.

On passe ensuite à la deuxième étape de planification $(k=1)$ que l'on effectue sur la base des données actualisées de $1^{\prime}$ horizon $H P^{I}$ commençant à la période $(T+P a)$, avec $H P^{I}=\{1+P a, 2+P a$, $\ldots, T+P a\}$. La deuxième itération de planification prend en compte les décisions prises à l'étape de planification précédente telles que les décisions gelées à l'itération $k=0$ sont reportées sans modifications à la deuxième itération de planification. Cette procédure itérative prend fin lorsque le nombre maximum d'itérations $(k \max )$ est atteint, avec: $k \max =\frac{(H D-T)}{P a}+1$ où $H D$ est le nombre de périodes sur lesquelles la demande est supposée connue.

\section{Approche par satisfaction de contraintes}

La procédure de planification dynamique énoncée en section 2 est formalisée sous la forme d'un problème de satisfaction de contraintes (Constraint Satisfaction Problem, CSP). Ainsi, cette partie rappelle les notions de base des problèmes de satisfaction de contraintes, les mécanismes de résolution et les extensions dans un contexte dynamique. 


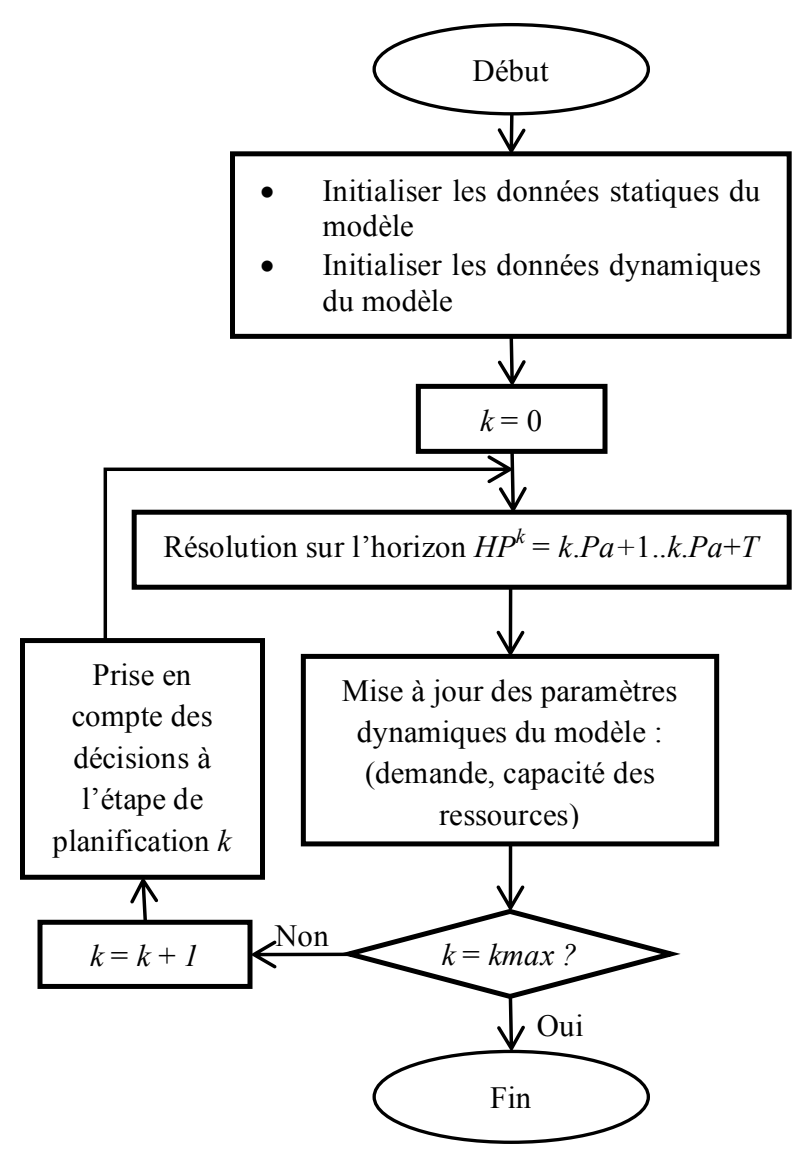

Figure 2. Approche proposée

\subsection{Problème de satisfaction de contraintes}

Un problème de satisfaction de contraintes (CSP) est défini par un ensemble de variables, chacune associée à un domaine de valeurs, et d'un ensemble de contraintes qui mettent en relation les variables. De façon plus formelle (Montanari, 1974; Mackworth, 1977; Tsang, 1993), on définit un CSP par un triplet $(\mathrm{X}, \mathrm{D}, \mathrm{C})$ tel que :

- $\quad X=\left\{X_{1}, X_{2}, \ldots, X_{n}\right\}$ est l'ensemble des variables du problème ;

- $\quad D=\left\{D_{1}, D_{2}, \ldots, D_{n}\right\}$ est l'ensemble des domaines. A chaque variable $X_{i} \in X, 1 \leq i \leq n$, est associé un domaine $D_{i} \in D$ qui représente l'ensemble des valeurs possibles pour $X_{i}$;

- $\quad C=\left\{C_{1}, C_{2}, \ldots, C_{m}\right\}$ est l'ensemble des contraintes où une contrainte $C_{i}$ est définie sur un ensemble de variables. 
Étant donné un CSP $(X, D, C)$, sa résolution consiste à affecter des valeurs aux variables de telle sorte que toutes les contraintes soient simultanément satisfaites. Cette résolution est basée sur des techniques de propagation de contraintes (phase de filtrage : réduction de l'espace de recherche en éliminant les valeurs des variables qui n'ont aucune chance d'intervenir dans une solution (Bessière et Régin, 2001 ; Zhang et Yap, 2001 ; Bessière et al., 2005) et sur une stratégie de recherche arborescente (phase de recherche de solutions : énumération des combinaisons de valeurs compatibles entre elles au regard de toutes les contraintes (Real-Full-Look-Ahead, Forward-Checking (Haralick et Elliot, 1980 ; Nadel, 1989), Maintaining Arc-Consistency (Sabin et Freuder, 1994)).

En dépit des avantages qu'il offre sur le plan de l'expressivité et de la modélisation des contraintes, le modèle CSP rencontre néanmoins des limites en présence de données soumises à des perturbations provenant de l'environnement ou d'autres agents. Un formalisme étendu, celui des DCSP (Dynamic Constraint Satisfaction Problem) permet d'adapter un CSP aux différentes perturbations qui peuvent survenir (Dechter et Dechter, 1988).

\subsection{Problème de satisfaction de contraintes dynamiques}

Dans l'objectif de répondre aux problèmes de perturbations qui caractérisent certaines données, notre structure décisionnelle a été modélisée sous la forme d'un problème de satisfaction de contraintes dynamiques. Un problème de satisfaction de contraintes dynamiques est une suite $P_{0}$, $P_{1}, \ldots$ de CSP classiques, tels que deux problèmes successifs $P_{i}$ et $P_{i+l}$ ne diffèrent que par l'ajout ou le retrait de certaines contraintes.

Comme nous l'avons vu dans le paragraphe précédent, un DCSP est une suite de CSP statiques. Pour trouver une solution, on peut résoudre chaque CSP indépendamment des autres à l'aide des méthodes de résolution classiques. Cependant, cette méthode de résolution passe à côté de la spécificité des DCSP : le caractère incrémental de la séquence de CSP. Ainsi, (Richaud, 2009) indique que Résoudre un DCSP revient à trouver une solution au CSP le plus récent (le dernier de la séquence). Du fait du caractère incrémental de la séquence, les solutions des autres CSP de la séquence peuvent être des solutions intermédiaires intéressantes. Ainsi l'efficacité des DCSP reposent sur l'utilisation de méthodes permettant d'exploiter efficacement le travail effectué aux étapes antérieures.

Essentiellement, deux approches distinctes apparaissent dans la littérature pour la résolution des DCSP. Les approches basées sur la mémorisation et la réutilisation des solutions et celles basées sur la mémorisation et la réutilisation du raisonnement.

\section{- L'approche par réutilisation du raisonnement}

Elle consiste à mémoriser des contraintes qui sont produites lors de la résolution du problème $P_{i}$ (contraintes induites) et qui sont des conséquences des contraintes explicites de $P_{i}$ et à les réutiliser dans la recherche courante pour la résolution du problème $P_{i+1}$. Cette approche regroupe plusieurs méthodes qui ne diffèrent l'une de l'autre que par le type des informations stockées avec chaque contrainte induite. Nous pouvons citer les méthodes basées sur les justifications et les méthodes basées sur les explications (Verfaillie et Jussien, 2005). La principale différence entre les deux méthodes est la nature de l'information enregistrée avec chaque contrainte induite. Alors que 
les justifications ne prennent en compte que la contrainte impliquée directement lors du retrait, les explications remontent jusqu'aux décisions responsables.

\section{- L'approche par réutilisation de solutions}

Cette approche repose sur l'idée suivante : si $S_{i}$ est une solution du problème $P_{i}$, alors une solution du problème $P_{i+1}$ (qui est proche de $P_{i}$ ) sera proche de $S_{i}$. Elle consiste à mémoriser et à réutiliser des solutions de problèmes précédents. Si un problème $P_{i}$ a été résolu et prouvé consistant, une solution $S_{i}$ de $P_{i}$ a été trouvée et enregistrée, une modification est survenue dans la définition du problème $P_{i}$ créant le problème $P_{\mathrm{i}+l}$, alors $S_{i}$ est aussi une solution de $P_{i+1}$ si $P_{i+1}$ est une relaxation de $P_{i}$, sinon $\left(P_{i+1}\right.$ est une restriction de $\left.P_{i}\right) S_{i}$ peut ne plus être une solution. L'hypothèse sur laquelle repose l'approche par réutilisation de solution (si une solution $S$ a été trouvée pour un problème $P$ et que le problème $P^{\prime}$ est proche du problème $P$ alors une solution $S^{\text {' }}$ de $P^{\prime}$ doit se trouver dans le voisinage de $S$ ), peut en fonction des situations, ne peut pas se vérifier et être totalement inefficace. L'approche par réutilisation de solutions regroupe plusieurs méthodes parmi lesquelles on trouve la recherche arborescente, la recherche locale et la méthode par relaxation-affectation de variables.

L'approche par réutilisation de solutions présente le double avantage d'être stable et efficace (Bellicha, 1993 ; Wallace et Grimes, 2010). Étant donné que la stabilité de la solution est un critère d'une importance prépondérante dans le domaine de la planification de la production, nous avons opté pour cette approche par réutilisation de solutions. Selon Herrera et Thomas (2010), la stabilité (nervosité) d'un PDP est relative aux différences entre les quantités planifiées recalculées à chaque itération du processus. Celui-ci sera stable si celles-là sont peu importantes et instable dans le cas contraire.

\subsection{Procédure de résolution}

Nous associons à chaque itération de planification $(k)$ et sur un horizon de planification $H P$, un CSP $P^{k}$. Les expérimentations sont effectuées à l'aide du logiciel Eclipse 6.0 qui est une extension du langage Prolog incluant des bibliothèques de programmation par contraintes.

Dans ce cadre, deux méthodes de résolution sont employées. Nous décrivons dans les paragraphes suivants, la méthode de résolution CSP statique et la méthode de résolution CSP dynamique.

\subsubsection{Procédure CSP statique}

Le problème étudié est un problème d'optimisation où l'objectif est donc de déterminer une solution associée à la minimisation d'une fonction objectif. Pour cela, nous utilisons une procédure de type séparation et évaluation (branch-and-bound). Cette méthode est basée sur une recherche arborescente d'une solution optimale dans un ensemble combinatoire de solutions possibles.

\subsubsection{Procédure CSP dynamique}

Nous décrivons ici la procédure de résolution dynamique. Dans ce cadre, nous adoptons l'approche par réutilisation de solution, décrite ci-dessus, pour la résolution des DCSP. Ainsi, la résolution d'un CSP $\left(P^{k}\right)$ est construite à partir de la solution relative au CSP $\left(P^{k-1}\right)$. Pour cela, 
nous utilisons les techniques de réparation et en particulier la technique de recherche locale dont le principe de base est le suivant :

- trouver une instanciation initiale pour toutes les variables du problème (leur donner des valeurs tentatives) ;

- trouver une contrainte en conflit (i.e., une contrainte qui ne satisfait pas les valeurs tentatives de ces variables), et instancier une variable de cette contrainte à sa nouvelle valeur (i.e., changer sa valeur tentative);

- instancier les variables restantes à leurs valeurs tentatives, lorsqu'il n'y a plus de contraintes en conflit.

Dans notre travail, l'instanciation initiale des variables d'un problème $\left(P^{k}\right)$ n'est autre que la solution du problème relative à l'itération de planification précédente $\left(P^{k-1}\right)$.

\section{Modélisation du processus de planification dynamique}

Ce modèle correspond au problème de planification à résoudre à chaque itération de planification $k$, sur l'horizon de planification $H P^{k}$. Dans ce modèle, la demande est supposée connue sur l'ensemble de l'horizon de planification et est mise à jour à chaque itération de planification. Nous détaillons ci-dessous les paramètres, les variables de décision et les contraintes de ce niveau.

\subsection{Paramètres}

$\{p\}=1 . . P:$ ensemble des produits finis $p$,

$\{s\}=1 . . S$ : ensemble des sites de production de la chaîne logistique,

$\left\{\right.$ Routage $\left._{p}\right\}$ : séquence des sites de production sur lesquels passe le produit $p$,

$k$ : numéro d'itération de la planification,

$T$ : nombre de périodes sur l'horizon de planification,

$H P$ : horizon de planification,

avec : $H P=\{1,2, \ldots, T\}$

$H G$ : horizon gelé associé à chaque type de décision,

avec : $H G=\{1,2, \ldots, D A\}$

où $D A$ est le délai d'anticipation de la décision

$P a(\tau)$ : pas de planification (nombre de périodes qui sépare deux itérations successives de planification),

$H D$ : nombre de périodes sur lesquelles la demande est supposée connue, avec: $H D=\left(k^{\max }\right.$ 1) $* P a+T$ et $k^{\max }=$ nombre maximum d'itérations de planification,

$L P p, L S p$ : délais d'obtention du produit fini $p$ lorsqu'il est respectivement fabriqué en interne ou sous-traité,

$S T_{p, t, s}$ : le volume de production maximal du produit $p$ en sous-traitance en période $t$ sur le site de production $s$,

$\operatorname{Hmax}_{s}$ : nombre maximal d'heures supplémentaires pour chaque site de production, 
$h_{p, s}$ : durée de fabrication unitaire d'un produit $p$ sur chaque site $s$,

$i_{p, s}, g_{p, s}, x_{p, s}, s_{p, s}, e_{s}$ : coût unitaire respectif de stockage, de rupture, de fabrication, de sous-traitance du produit $p$ sur le site $s$ et d'utilisation d'une heure supplémentaire sur un site $s$,

$C_{t, s}$ : capacité de production interne pour chaque site de production (nombre d'heures de travail disponibles),

$D_{p, t}^{k}$ : demande globale du produit fini $p$ pour la période $t$.

\subsection{Variables de décision à l'itération de planification $k$}

$X_{p, t, s}^{k}$ : quantité de produits $p$ lancés en production interne en période $t$ sur le site $s$,

$S_{p, t, s}^{k}$ : quantité de produits $p$ lancés en sous-traitance interne en période $t$ sur le site $s$,

$H_{t, s}^{k}$ : nombre d'heures supplémentaires utilisées en période $t$ sur le site $s$,

$I_{p, t, s}^{k}:$ niveaux de stock en fin de période $t$ pour le produit fini $p$ sur le site $s$,

$G_{p, t, s}^{k}:$ niveaux de rupture en fin de période $t$ pour le produit fini $p$ sur le site $s$

$B^{k}:$ variable binaire $=1$ si la contrainte d'activation est vraie en période $t, 0$ sinon.

\subsection{Contraintes}

$$
\begin{aligned}
& I_{p, t, s}^{k}-G_{p, t, s}^{k}=I_{p, t-1, s}^{k}-G_{p, t-1, s}^{k}+X_{p, t-L P, s}^{k}+S_{p, t-L S, s}^{k}-D_{p, t}^{k} \\
& \forall p, \forall s \in \text { Routage }_{p}, \forall t \in H P^{k} \\
& \sum_{p=1}^{P} h_{p, s} * X_{p, t, s}^{k} \leq C_{t, s}+H_{t, s}^{k} \quad \forall p, \forall s \in \text { Routage }_{p}, \forall t \in H P^{k} \\
& S_{p, t, s}^{k} \leq S T_{p, t, s} \quad \forall p, \forall s \in \text { Routage }_{p}, \forall t \in H P^{k} \\
& H_{t, s}^{k} \leq \operatorname{Hmax}_{s} \quad \forall s \in \text { Routage }_{p}, \forall t \in H P^{k} \\
& \sum_{p=1}^{P} h_{p, s} * D_{p, t}^{k}>C_{t, s}^{k}+H_{t, s}^{k} \Rightarrow S_{p, t-L S, s}^{k}>0 \\
& \forall p, \forall s \in \text { Routage }_{p}, \forall t \in H P^{k} \\
& X_{p, t, s}^{k+1}=X_{p, t+P a, s}^{k} \quad \forall p, \forall s \in \text { Routage }_{p}, \forall t \in H P^{k} \\
& S_{p, t, s}^{k+1}=S_{p, t+P a, s}^{k} \quad \forall p, \forall s \in \text { Routage }_{p}, \forall t \in H P^{k} \\
& H_{t, s}^{k+1}=H_{t+P a, s}^{k} \quad \forall p, \forall s \in \text { Routage }_{p}, \forall t \in H P^{k}
\end{aligned}
$$




\subsection{Fonction objectif}

$$
\begin{gathered}
\min \sum_{t=0}^{T-1}\left[\sum _ { p = 1 } ^ { P } \left[\sum_{s \in \text { Routage }_{p}} i_{p, s} * I_{p, t, s}^{k}+g_{p, s} *\right.\right. \\
\left.\left.e_{s} * H_{s, t}^{k}\right]\right]
\end{gathered} \quad \begin{gathered}
G_{p, t, s}^{k}+x_{p, s} * X_{p, t, s}^{k}+s_{p, s} * S_{p, t, s}^{k}+ \\
\forall k
\end{gathered}
$$

La contrainte (1) garantit la conservation de la matière. La contrainte (2) permet de vérifier que la capacité de production interne augmentée des heures supplémentaires est compatible avec la charge globale de production que doit assurer le site. Les contraintes (3) et (4) assurent le respect du volume de production maximal du sous-traitant et le volume d'heures supplémentaires utilisées qui ne doit pas dépasser la valeur maximale autorisée sur chaque site de production. La contrainte (5) est une contrainte conditionnelle. Elle permet l'activation ou la désactivation de l'action de sous-traitance. Les contraintes (6) à (8) formalisent la cohérence des décisions entre deux itérations de planification successives $(k)$ et $(k+1)$ et ce, pour toutes les décisions (production en interne, sous-traitance et allocation d'heures supplémentaires). La fonction objectif (9) minimise la somme de tous les coûts sur tous les sites de production et sur toutes les périodes de l'horizon de planification $H P$.

\section{Analyse de performance : démarche méthodologique}

Cette section porte sur la méthodologie développée pour analyser la performance du modèle de planification dynamique d'une chaîne logistique. Dans cette démarche, nous associons à chaque itération de planification $k$ un $\operatorname{CSP}\left(P^{k}\right)$ correspondant au problème de planification à résoudre. Le problème $\left(P^{k+1}\right)$ constitue une actualisation du problème $\left(P^{k}\right)$ par l'ajout ou le retrait de certaines contraintes issues de perturbations internes ou externes (figure 3 ).

Pour une actualisation donnée CSP $\left(P^{k+1}\right)$, nous appliquons, séparément, deux méthodes de résolution différentes: une méthode de résolution statique et une méthode de résolution dynamique. La performance d'une solution est renseignée par l'ensemble des mesures de la stabilité aux niveaux sites. Dans la suite, nous exposons les objectifs de l'analyse et l'approche comparative. Nous présentons ensuite l'indicateur de stabilité formalisé.

\subsection{Objectifs de l'analyse : mesurer la stabilité}

La performance d'une chaîne logistique est évaluée par l'ensemble des mesures de satisfaction des objectifs que se fixent les entreprises partenaires. Dans notre travail, les résultats numériques obtenus par différentes simulations sont comparés sur la base d'indicateurs de stabilité localement au niveau «Site». Ces mesures portent sur la quantification de la réorganisation induite par la modification d'un programme de production. Une solution est alors évaluée par la comparaison entre : 


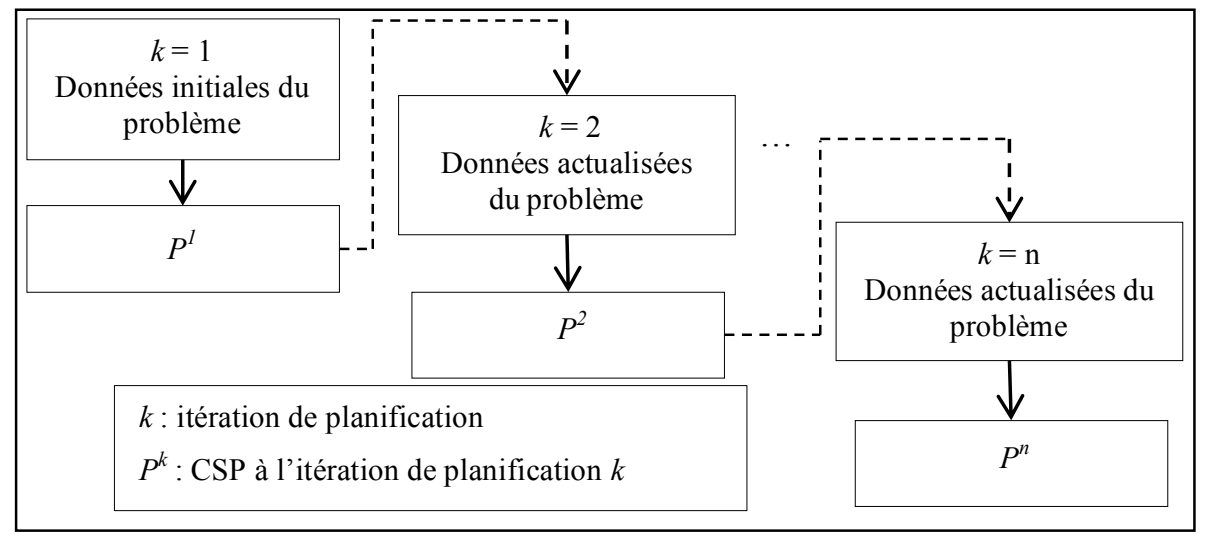

Figure 3. Schéma d'actualisation d'un problème de planification

- les décisions engagées, correspondantes au déroulement réel et prévu des activités de transformation du flux physique, et

- les décisions du plan modifié, visant à réorganiser et à mettre à jour ces mêmes activités suite à un aléa.

Pour une solution donnée, les résultats traduisent l'étendue du changement sur les décisions de planification (quantités à produire). Ces résultats sont à la base du référentiel de mesure de performance utilisé dans la suite de ce travail pour évaluer la stabilité d'une solution, notion que nous définissons ci-dessous.

\subsection{La notion de stabilité : définitions}

Durieux et Pierreval (2003) indiquent que le terme stabilité est généralement mis en opposition à celui de nervosité. Stabilité et nervosité de la planification sont souvent traitées dans la littérature au niveau du calcul des besoins (MRP) (Yano et Carlson, 1987 ; Ho, 1989 ; Sridharan et Laforge, 1990 ; Jensen, 1993 ; Heisig, 1998). Calculer un programme directeur de production (PDP) revient à mettre en place un horizon glissant, ce qui implique de le recalculer avec une périodicité $\Delta t$; par conséquent, ce type de pratique induit des variations dans les quantités de production résultantes. Selon (Herrera et Thomas, 2010), la stabilité (nervosité) d'un PDP est alors relative aux différences entre les quantités planifiées recalculées à chaque itération du processus. Celui-ci sera stable si celles-là sont peu importantes et instable dans le cas contraire. Pour Vollman et al., (1997), la nervosité est communément définie comme des changements significatifs dans les plans $M R P$, qui ont lieu même en cas de changement minime du niveau supérieur ou du PDP. Les changements concernent les quantités ou les périodes des ordres planifiés. Il ressort que lorsque nous nous intéressons à la stabilité d'un système, nous cherchons à ne pas remettre en cause très souvent les décisions que nous aurions prises. 
Dans notre travail, nous abordons la notion de stabilité en tant qu'aptitude d'une solution de planification/ordonnancement à assurer un certain niveau de continuité du programme de production initial face aux variations de certaines données (demande, capacité, etc.). Cette notion est employée pour compter le nombre de variables modifiées par une variation d'une donnée, révélant ainsi la plus ou moins grande aptitude d'une solution à assurer la performance de stabilité.

\subsection{Indicateur de performance}

\subsubsection{Protocole d'évaluation de la performance}

La démarche générale est basée sur l'évaluation de la performance suite à deux itérations successives telles que : la première itération $(k)$ correspond au problème initial et la deuxième itération $(k+1)$ correspond au même problème avec prise en compte d'aléas. La solution initiale (relative au problème initial) que nous notons par $\alpha^{k}$ est obtenue par la méthode de résolution statique. La nouvelle solution (relative au problème avec prise en compte d'aléas) que nous notons par $\alpha^{k+1}$ est obtenue, distinctement et à des fins de comparaison, suivant les deux approches de résolution statique et dynamique.

L'approche comparative est menée en considérant une variation de la demande. Nous simulons différents taux ( $20 \%$ de modifications, $40 \%$ de modifications, etc.). Pour évaluer l'étendue de la perturbation possible sur les activités de production, nous comparons les deux nouvelles solutions obtenues, distinctement par résolution statique et par résolution dynamique, par rapport à la solution initiale. Ainsi, nous introduisons un indicateur $P_{S}^{N}$ qui correspond à la distance de perturbation.

\subsubsection{Notion de distance}

Pour rappel, un DCSP $\Theta$ est une suite $P^{0}, P^{l}, \ldots$ de CSP classiques, tels que deux problèmes successifs $P^{k}$ et $P^{k+1}$ ne diffèrent que par l'ajout ou le retrait de certaines contraintes.

Soit $\alpha^{k}$ une solution à $P^{k}$ et $\alpha^{k+1}$ une solution à $P^{k+1}$. Nous définissons $\delta^{1}$ comme l'ensemble de variables $v$ tel que : l'instanciation $h$ de $v$ dans $\alpha^{k}$ est différente de l'instanciation $h$ ' de $v$ dans $\alpha^{k+1}$ :

$$
\delta^{1}\left(\alpha^{k}, \alpha^{k+1}\right)=\left\{v \in \Theta \mid \frac{v}{h} \in \alpha^{k} \& \frac{v}{h^{\prime}} \in \alpha^{k+1} \& h \neq h^{\prime}\right\}
$$

Ainsi, la première fonction $P_{S}^{N}$ est définie par le cardinal de l'ensemble. Elle correspond à la distance de perturbation, connue dans la littérature mathématique comme la distance de Hamming :

$$
P_{S}^{N}\left(\alpha^{k}, \alpha^{k+1}\right)=\left|\delta^{1}\left(\alpha^{k}, \alpha^{k+1}\right)\right|
$$

Le plan de production est caractérisé par des variables relatives à la planification des produits comme le volume de production interne, le volume de production sous-traitée, les stocks, les arriérés, etc. L'indicateur de performance que nous employons dans notre travail relève du niveau énuméré ci-dessus et en particulier les volumes de production. 


\subsubsection{Indicateur de stabilité : distance moyenne de perturbation}

La gestion d'une chaîne logistique nécessite la disposition d'informations sur l'état courant des différents sites de production. Ainsi, la définition d'une solution de planification retenue pour la chaîne logistique, et son évaluation sur le niveau local des sites de production constitue un moyen d'assurer un certain niveau de stabilité.

Pour le premier niveau de décision correspondant à la planification, la distance moyenne de perturbation $\left(P_{S}^{N}\right)$ pour un site donné est calculée pour les variables $\left(X_{p, t, s}\right)$ correspondant aux quantités des produits $p$ lancés en production interne sur les différentes périodes $t$ sur le site $s$. Elle est définie par la formule suivante :

$$
P_{S}^{N}\left(\alpha^{k}, \alpha^{k+1}\right)=\frac{\left|\delta^{1}\left(\alpha^{k}, \alpha^{k+1}\right)\right|}{P_{S}^{* T}}
$$

avec :

$$
\delta^{1}\left(\alpha^{k}, \alpha^{k+1}\right)=\left\{X_{p, t s} \in \Theta \mid X_{p, t, s} / h \in \alpha^{k} \& X_{p, t, s} / h^{\prime} \in \alpha^{k+1} \& h \neq h^{\prime}\right\}
$$

$P_{s}:$ nombre de produits traversant le site $s, T:$ nombre de périodes

\section{Expérimentation numérique et évaluation de l'approche}

Cette section porte sur l'analyse du modèle de planification dynamique et l'évaluation des deux approches de résolution CSP Statique/CSP Dynamique, et leurs impacts sur la conduite d'une chaîne logistique. Le cadre est un cas d'étude de réseau de sites de production. Le problème, de nature académique, est choisi de complexité réduite pour éviter une inutile explosion des données et une difficulté d'analyse des résultats d'expérimentation.

\subsection{Présentation du cas d'étude}

La chaîne logistique se compose de plusieurs sites de production appartenant à une même entreprise, et réalisant différents types de produits. La réalisation d'un produit nécessite une succession d'opérations de fabrication, réalisée chacune sur un site donné. Il s'agit d'organiser la production de 5 produits finis $(A, B, C, D$ et $E$ ), sur un horizon de planification de 16 périodes. Le profil de la demande prévisionnelle relatif à la première itération de planification est présenté dans le tableau 1. Les 5 produits sont réalisés sur les différents sites de production (5 sites) selon les gammes indiquées sur la figure 4. Chaque site est défini par sa capacité de production (en nombre de machines). Dans un souci de simplification, nous considérons que les différents sites utilisent des machines parallèles. 


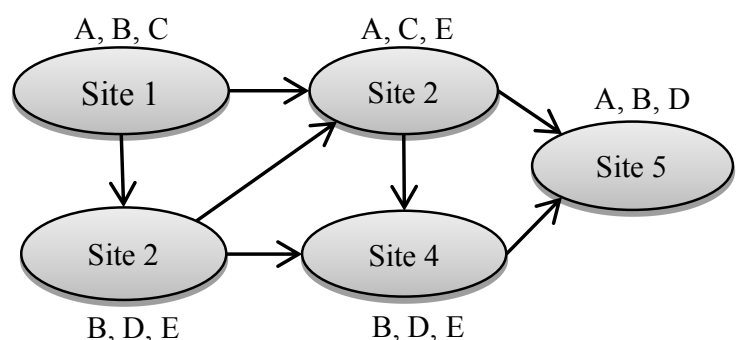

Figure 4. Gamme de fabrication des différents produits

Tableau 1. Profil de la demande (première itération de planification : $k=1$ )

\begin{tabular}{|c|c|c|c|c|c|c|c|c|c|}
\hline & $t$ & 1 & 2 & 3 & 4 & 5 & 6 & 7 & 8 \\
\hline \multirow{6}{*}{ 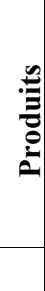 } & $\mathrm{A}$ & 42 & 90 & 62 & 43 & 49 & 50 & 68 & 95 \\
\hline & B & 68 & 85 & 73 & 56 & 50 & 60 & 48 & 55 \\
\hline & C & 55 & 43 & 92 & 33 & 35 & 75 & 50 & 68 \\
\hline & $\mathrm{D}$ & 60 & 68 & 59 & 50 & 52 & 40 & 80 & 63 \\
\hline & $\mathrm{E}$ & 48 & 35 & 63 & 71 & 60 & 32 & 65 & 75 \\
\hline & $t$ & 9 & 10 & 11 & 12 & 13 & 14 & 15 & 16 \\
\hline \multirow{5}{*}{ 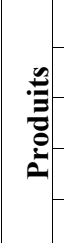 } & A & 86 & 50 & 90 & 62 & 43 & 43 & 49 & 50 \\
\hline & B & 63 & 51 & 72 & 68 & 53 & 59 & 50 & 52 \\
\hline & C & 59 & 60 & 35 & 75 & 50 & 71 & 60 & 32 \\
\hline & $\mathrm{D}$ & 82 & 43 & 42 & 90 & 62 & 48 & 35 & 63 \\
\hline & $\mathrm{E}$ & 53 & 56 & 59 & 50 & 52 & 42 & 90 & 62 \\
\hline
\end{tabular}

\subsection{Problématique et scénarios considérés}

La chaîne logistique considérée s'inscrit dans le cadre d'un réseau de sites au sein duquel il existe un service logistique central assurant la planification et l'ordonnancement agrégé des charges de fabrication. Il s'agit de répartir et de coordonner les productions entre les sites tout en laissant à chaque site une autonomie au niveau de l'ordonnancement détaillé local.

Différents phénomènes pratiques peuvent être explorés sur la base de ce cas d'étude. En effet, après avoir planifié la production sur les sites et engagé leur organisation sur un horizon donné, le service logistique central peut se retrouver par exemple, à une période $t$, face à une annulation imprévue d'une commande client pour un ou plusieurs produits, une chute de la capacité de production sur un ou plusieurs sites suite à des pannes machines ou grèves d'employés ou encore un retard d'approvisionnements nécessaires à la production d'un ou plusieurs produits engendrant une re-planification de ces quantités à la période suivante $t+1$. Pour ces différents scénarios, le 
service logistique central sera amené à réorganiser la solution initiale avec le minimum de perturbation de la production engagée sur les différents sites et du planning annoncé aux différents intervenants.

Dans ce cadre, nous menons deux séries d'expérimentations différentes qui correspondent respectivement à une variation de la demande et une variation de la capacité. Le taux de variation est considéré comme un scénario donné et non pas une grandeur de la variation. L'objectif est d'évaluer et de comparer la stabilité fournie par la résolution CSP statique et la résolution CSP dynamique face au même type et à la même taille d'une variation simulée.

\section{3. Évaluation de la stabilité au niveau site de production}

\subsubsection{Expérimentation avec une variation de la demande}

\subsubsection{Scénario d'expérimentation}

Pour une période donnée $(t=11)$, la variation porte sur une chute de la demande. Cette simulation porte progressivement et cumulativement sur le produit A, puis produit B jusqu'au produit D. Le taux de variation est égal au nombre de produits variés sur le nombre total de produits. Nous nous sommes limités à un taux de variation égal à $80 \%$ (4 produits variés sur 5). Sur chacun des produits la variation de la demande est d'une quantité égale à (-10).

- Taux de variation $20 \%$ : Produit $\mathrm{A}$ de 90 à 80 ;

- Taux de variation $40 \%$ : Produit $\mathrm{A}$ de 90 à 80, et Produit B de 72 à 62 ;

- Taux de variation $60 \%$ : Produit $\mathrm{A}$ de 90 à 80, et Produit $\mathrm{B}$ de 72 à 62, et Produit $\mathrm{C}$ de 35 à 25 ;

- Taux de variation $80 \%$ : Produit $\mathrm{A}$ de 90 à 80 , et Produit $\mathrm{B}$ de 72 à 62, et Produit $\mathrm{C}$ de 35 à 25 , et Produit $\mathrm{D}$ de 42 à 32.

Pour toutes ces mesures de variations, le taux de variation est considéré comme un scénario donné et non pas une grandeur de la variation.

\subsubsection{Résultats expérimentaux}

Nous présentons une vue détaillée des résultats obtenus avec une expérimentation basée sur une variation de la demande ainsi que les résultats dans le cadre d'une résolution statique et d'une résolution dynamique.

Le tableau 2 détaille les résultats numériques. À partir de ce tableau, nous établissons les constatations suivantes :

- Pour la résolution statique et pour chaque site de production, l'indicateur de performance (stabilité $P_{S}^{N}$ ) évolue dans le même sens. En effet, la distance de perturbation évolue en croissance avec l'évolution du taux de variation.

- Pour la résolution dynamique, l'indicateur de performance tend vers zéro indépendamment du taux de variation. 
- Avec la méthode de résolution statique, nous constatons que la distance de perturbation varie entre 0,39 et 0,44 . Il n'y a pas de grande différence entre les valeurs de l'indicateur de performance d'un site à un autre.

- Pour un même taux de variation, l'indicateur de performance évolue indépendamment en se déplaçant d'un site à un autre et cela est vrai pour la résolution statique et pour la résolution dynamique.

Tableau 2. Résultats numériques dans le cas d'une variation de la demande

\begin{tabular}{|c|c|c|c|c|c|c|c|c|c|c|c|}
\hline \multirow{6}{*}{ 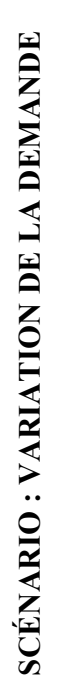 } & 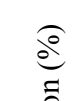 & \multicolumn{5}{|c|}{$\begin{array}{l}\text { Indicateur de stabilité } \\
\text { Résolution statique }\end{array}$} & \multicolumn{5}{|c|}{$\begin{array}{l}\text { Indicateur de stabilité } \\
\text { Résolution dynamique }\end{array}$} \\
\hline & $\stackrel{. \frac{\pi}{\tilde{U}}}{>}$ & 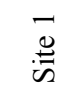 & 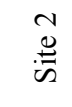 & $\stackrel{m}{\stackrel{m}{0}}$ & $\stackrel{+}{\stackrel{\oplus}{厶}}$ & $\stackrel{n}{\stackrel{0}{*}}$ & 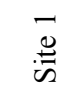 & 曽 & $\stackrel{m}{\stackrel{m}{*}}$ & $\stackrel{+}{\stackrel{\oplus}{\omega}}$ & $\stackrel{n}{\infty}$ \\
\hline & $20 \%$ & 0,44 & 0,43 & 0,44 & 0,43 & 0,39 & 0,02 & 0 & 0,02 & 0 & 0,02 \\
\hline & $40 \%$ & 0,44 & 0,02 & 0,44 & 0,43 & 0,39 & 0 & 0 & 0 & 0 & 0 \\
\hline & $60 \%$ & 0,44 & 0,43 & 0,44 & 0,43 & 0,39 & 0 & 0 & 0 & 0 & 0 \\
\hline & $80 \%$ & 0,44 & 0,44 & 0,44 & 0,44 & 0,41 & 0 & 0 & 0 & 0 & 0 \\
\hline
\end{tabular}

\subsubsection{Expérimentation avec une variation de la capacité}

\subsubsection{Scénario d'expérimentation}

La deuxième série d'expérimentations porte sur une variation de la capacité des sites de production. Pour la période $t=13$, cette simulation porte progressivement et cumulativement sur le site 1 , puis site 2 jusqu'au site 4 . Sur chacun des sites, la variation de la capacité est d'une quantité égale à (-1). Le taux de variation est égal au nombre de sites variés sur le nombre total de sites.

- Taux de variation $20 \%: \mathrm{S} 1$ de 5 à 4 ;

- Taux de variation $40 \%: \mathrm{S} 1$ de 5 à 4 , et $\mathrm{S} 2$ de 4 à 3 ;

- Taux de variation $60 \%: \mathrm{S} 1$ de 5 à 4 , et $\mathrm{S} 2$ de 4 à 3 , et $\mathrm{S} 3$ de 4 à 3 ;

- Taux de variation $80 \%: \mathrm{S} 1$ de 5 à 4 , et $\mathrm{S} 2$ de 4 à 3, et $\mathrm{S} 3$ de 4 à 3, et $\mathrm{S} 4$ de 3 à 2.

Pour toutes ces mesures de variations, le taux de variation est considéré comme un scénario donné et non pas une grandeur de la variation. 


\subsubsection{Résultats expérimentaux}

Nous présentons une vue détaillée des résultats obtenus avec une expérimentation basée sur une variation de la capacité ainsi que les résultats dans le cadre d'une résolution statique et d'une résolution dynamique. Le tableau 3 détaille les résultats numériques.

Tableau 3. Résultats numériques dans le cas d'une variation de la capacité

\begin{tabular}{|c|c|c|c|c|c|c|c|c|c|c|c|}
\hline \multirow{6}{*}{ 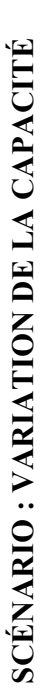 } & $\overbrace{\tilde{d}}^{e}$ & \multicolumn{5}{|c|}{$\begin{array}{c}\text { Indicateur de stabilité } \\
\text { Résolution statique }\end{array}$} & \multicolumn{5}{|c|}{$\begin{array}{l}\text { Indicateur de stabilité } \\
\text { Résolution dynamique }\end{array}$} \\
\hline & $\begin{array}{l}\stackrel{\pi}{\pi} \\
\overbrace{}^{\pi}\end{array}$ & $\stackrel{\bar{\omega}}{\mathscr{\omega}}$ & $\stackrel{\sim}{\stackrel{\sim}{\infty}}$ & $\stackrel{n}{\stackrel{n}{n}}$ & $\stackrel{+}{\stackrel{\oplus}{0}}$ & $\stackrel{n}{\stackrel{n}{n}}$ & $\stackrel{\vec{g}}{\text { is }}$ & 光 & $\stackrel{n}{\infty}$ & $\stackrel{+}{\stackrel{\Delta}{*}}$ & 㤎 \\
\hline & $20 \%$ & 0,43 & 0,37 & 0,44 & 0,41 & 0,39 & 0 & 0 & 0 & 0 & 0 \\
\hline & $40 \%$ & 0,43 & 0,46 & 0,44 & 0,41 & 0,39 & 0 & 0 & 0 & 0 & 0 \\
\hline & $60 \%$ & 0,43 & 0,46 & 0,5 & 0,41 & 0,46 & 0 & 0 & 0 & 0 & 0 \\
\hline & $80 \%$ & 0,43 & 0,46 & 0,44 & 0,33 & 0,39 & 0 & 0 & 0 & 0 & 0 \\
\hline
\end{tabular}

Nous retrouvons des éléments similaires par rapport à l'expérimentation précédente. Nous notons que :

- Pour la résolution statique, sur chaque site de production, l'indicateur de stabilité $P^{N}$ est en quasi-constance compte tenu de l'évolution du taux de la variation.

- Pour la résolution dynamique, l'indicateur de performance tend vers zéro indépendamment du taux de la variation.

- Avec la résolution statique, nous constatons que la stabilité $P^{N}$ varie entre 0,39 et 0,47 . Il n'y a pas de grande différence entre les valeurs des indicateurs de performance d'un site à un autre.

- Pour un même taux de variation, l'indicateur de performance évolue indépendamment en se déplaçant d'un site à un autre et cela est vrai pour la résolution statique et pour la résolution dynamique.

\subsubsection{Interprétation des résultats}

En examinant les résultats pour les deux types de variation (demande et capacité), nous établissons les conclusions suivantes : pour les deux types de variations considérées, la résolution dynamique offre une stabilité meilleure que la résolution statique ; 
Avec la résolution dynamique, l'indicateur de stabilité tend vers zéro et ceci pour les deux séries d'expérimentations. Ceci peut s'expliquer par la stratégie de résolution que nous avons adoptée. En effet, l'idée était de partir de la solution précédente pour la réparation du problème ayant subi des perturbations. Pour cela, nous avons choisi de réparer les contraintes en conflit en augmentant progressivement les valeurs des variables $\left(X_{p, \mathrm{~s}, \mathrm{t}}\right)$ d'une valeur égale à 1 . Par la suite, la solution est très proche de la solution initiale. Afin de satisfaire ces objectifs de stabilité, la résolution tolère la régulation sur d'autres variables. Par exemple, si nous considérons le cas de la variation de la demande avec un taux de variation égal à $20 \%$, la demande en produit 1 sur la période $(t=13)$, est passé de 90 à 80 et la solution obtenue par une résolution dynamique montre que la régulation a porté sur la quantité en stock $I_{p, s, t}$.

\subsection{Sensibilité de la fonction objectif face aux variations}

Dans la section précédente, nous avons évalué et comparé la stabilité de la solution par type de variation suivant la méthode de résolution. Nous nous intéressons ici à l'évaluation du coût de production pour la méthode de résolution statique et la méthode de résolution dynamique et pour chaque type de variation (figures 5 et 6 ).

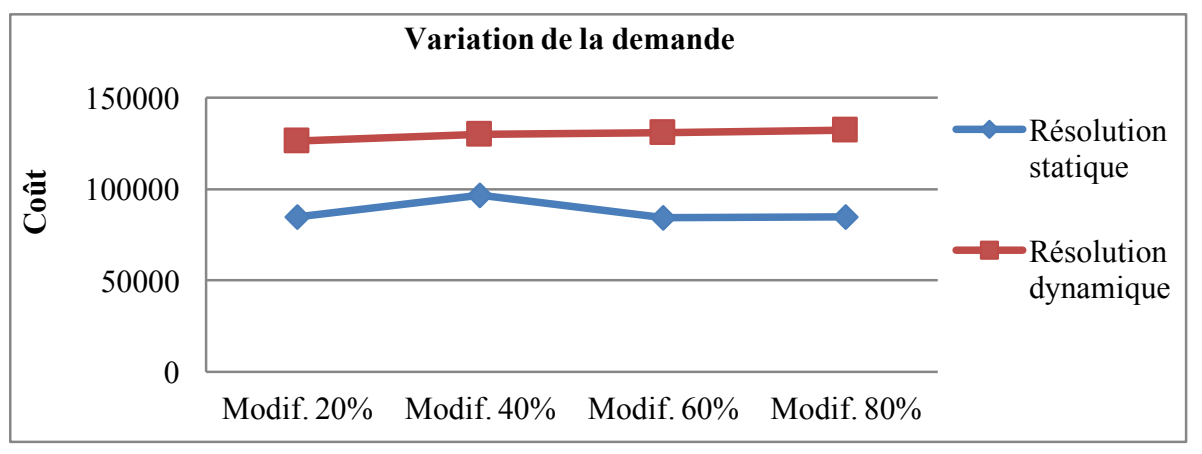

Figure 5. Évaluation du coût dans le cas d'une variation de la demande

Dans les deux expérimentations, le coût calculé avec la méthode de résolution dynamique est plus élevé. Ceci est dû à la stratégie de recherche dynamique que nous avons choisie qui opte pour une solution plus stable sur le niveau de la planification de la production. En effet, dans le but d'avoir un plan de production le moins perturbé possible, les réparations des solutions ont porté davantage sur les stockages et les heures supplémentaires qui ont un coût plus élevé, d'où le coût global plus élevé avec la méthode de résolution dynamique. 


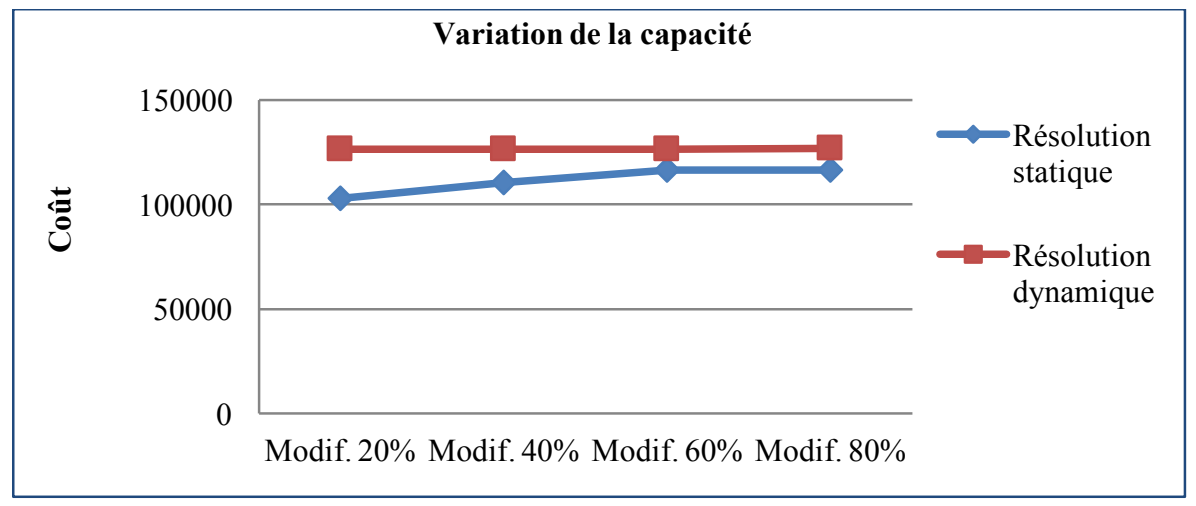

Figure 6. Évaluation du coût dans le cas d'une variation de la capacité

\subsection{Temps de résolution}

L'implémentation a été effectuée sous $\mathrm{ECL}^{i} \mathrm{PS}^{e} 6.0$ qui est une extension du langage Prolog incluant des bibliothèques de programmation par contraintes (voir http://eclipseclp.org/). Les simulations ont été effectuées sur un ordinateur portable équipé d'un processus Intel ${ }^{\circledR}$ Core ${ }^{\mathrm{TM}} \mathrm{i}$ 2450M CPU cadencé à $2.5 \mathrm{Ghz}$.

Pour chaque scénario étudié, nous présentons dans le tableau 4, les temps de résolution obtenus avec les deux méthodes de résolution considérées. Nous pouvons constater que les temps de résolution obtenus en utilisant la méthode de résolution CSP dynamique sont relativement faibles par rapport à ceux obtenus en utilisant la méthode de résolution CSP statique. Cela montre que la méthode dynamique offre une meilleure performance non seulement au niveau de la stabilité de la solution mais aussi au niveau du temps de calcul.

\section{Conclusion}

Cet article propose une approche de planification dynamique à horizon glissant permettant d'optimiser les coûts globaux de la production. Dans le but de minimiser l'effet des perturbations engendrées par diverses incertitudes, la modélisation a été réalisée sous la forme d'un problème de satisfaction de contraintes dynamiques $(D C S P)$. Sur la base de ce formalisme, deux méthodes de résolution, statique et dynamique, ont été implémentées en exploitant les fonctionnalités d'un langage de programmation par contraintes. Une expérimentation a été menée sur la base d'un cas de planification de la production d'une chaîne logistique et a porté sur une variation de la demande et de la capacité.

La performance d'une solution de planification est renseignée par l'ensemble des mesures de la stabilité. Pour cela, nous avons développé un indicateur qui caractérise le plan établi au niveau planification. Bien que les perturbations évaluées ne soient pas d'une variabilité importante, les résultats expérimentaux montrent que la méthode de résolution dynamique offre de meilleures performances sur le plan de la stabilité de la solution. 
Tableau 4. Temps d'exécution

\begin{tabular}{|c|c|c|c|c|}
\hline \multirow{3}{*}{ 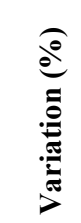 } & \multicolumn{4}{|c|}{ Temps de résolution (s cpu) } \\
\hline & \multicolumn{2}{|c|}{ Variation de la demande } & \multicolumn{2}{|c|}{ Variation de la capacité } \\
\hline & ésolution statiqu & solution dynamic & qésolution statiqu & olution dynami \\
\hline $20 \%$ & 10933,83 & 1031,06 & 17904,59 & 6144,54 \\
\hline $40 \%$ & 10021,75 & 835,10 & 14904,15 & 6076,25 \\
\hline $60 \%$ & 8662,49 & 830,92 & 16446,94 & 3539,23 \\
\hline $80 \%$ & 13406,38 & 835,63 & 13649,40 & 835,63 \\
\hline
\end{tabular}

D'autres perturbations portant sur d'autres aspects aideront à mieux caractériser les performances de l'approche dynamique. Il sera ainsi intéressant d'étudier l'influence d'autres paramètres liés à la planification comme le pas de planification, la longueur de l'horizon gelé, ou à la méthode de recherche de solutions. Une autre voie de recherche serait d'étudier des techniques de résolution dynamique alternatives, par exemple une procédure itérative exploitant la programmation linéaire en nombres entiers ou certaines métaheuristiques et d'analyser leur performance par rapport à la méthode de résolution par CSP dynamique.

\section{Bibliographie}

Al-Othman W.B.E., Lababidi H.M.S., Alatiqi I.M. and Al-Shayji K. (2008). Supply chain optimization of petroleum organization under uncertainty in market demands and prices. European Journal of Operational Research, 189, 822-840.

Amrani-Zouggar A. (2009). Impact des contrats d'approvisionnement sur la performance de la chaîne logistique : Modélisation et simulation. Thèse de doctorat, Université Bordeaux 1, France.

Bellicha A. (1993). Maintenance of solution in a dynamic constraint satisfaction problem. In Proceedings of applications of artificial intelligence in Engineering VIII, p. 261-274.

Bessière C. and Régin J.-C. (2001). Refining the basic constraint propagation algorithm. In Proceedings of the 17th International Joint Conference on Artificial Intelligence (IJCAI'01), Seattle, WA, USA, p. 309-315.

Bessière C., Régin J.-C., Yap R. and Zhang Y. (2005). An optimal coarse-grained arc consistency algorithm. Artificial Intelligence, 2(165), 165-185. 
Bihlmaier R., Koberstein A. and Obst R. (2009). Modeling and optimizing of strategic and tactical production planning in the automotive industry under uncertainty. OR Spectrum, 3: 311-336.

Davis T. (1993). Effective supply chain management. Sloan Management Review, 34: 35-46.

Dechter R. and Dechter A. (1988). Belief maintenance in dynamic constraints networks. In Proceedings of the Seventh National Conference on Artificial Intelligence (AAAI'88), p. 37-42.

Durieux-Paris S., Genin P. et Thierry C. (2007). Prise de décision dans la chaîne logistique en mode incertain. Document de synthèse du projet GDR MACS.

Durieux S. et Pierreval H. (2003). De la notion de robustesse dans les études de simulation (2003). Actes de la $4^{e}$ Conférence francophone de modélisation et simulation (MOSIM'03), Toulouse.

Fargier H., Lang J., Martin-Clouaire R. et Schiex T. (2002). Traitement de problèmes de décision sous incertitudes par des problèmes de satisfaction de contraintes. Revue d'intelligence artificielle, 11(3), 375-398.

Galbraith J. (1973). Designing complex organizations. Addison-Wesley, Massachusetts.

Génin P. (2003). Planification tactique robuste avec usage d'un APS : Proposition d'un mode de gestion par plan de référence. Thèse de Doctorat, École des Mines de Paris, France.

Haralick R. and Elliott G. (1980). Increasing tree search efficiency for constraint satisfaction problems. Artificial Intelligence, 14(3), 263-313.

Heisig G. (1998). Planning stability under (s,S) inventory control rules. OR Spektrum 20(4): 215-228.

Herrera C. et Thomas A. (2009). Un modèle pour limiter la nervosité et l'instabilité du programme directeur de production. $8^{\mathrm{e}}$ Congrès international de génie industriel, Bagnères de Bigorre, France.

Ho C. (1989). Evaluating the impact of operating environments on MRP system nervousness. International Journal of Production Research, 27, 1115-1135.

Jensen T. (1993). Measuring and improving planning stability of reorder point lot-sizing policies. International Journal of Production Economics, 30-31, 167-178.

Lalami M.E. (2012). Contribution à la résolution de problèmes d'optimisation combinatoire : méthodes séquentielles et parallèles. Thèse de doctorat, Université Paul Sabatier, Toulouse, France.

Lee H.L. and Billington C. (1993). Material management in decentralized supply chain. Operations Research, $41(5)$.

Lim S.J., Jeong S.J., Kim K.S. and Park M.W. (2006). A simulation approach for production-distribution planning with consideration given to replenishment policies. The International Journal of Advanced Manufacturing Technology, 27, 593-603.

Mackworth A.K. (1977). Consistency in networks of relations, Artificial Intelligence, 8(1), 99-118.

Minton S., Johnston M.D., Philips A.B. and Laird P. (1992). Minimizing conflicts: a heuristic repair method for constraint satisfaction and scheduling problems. Artificial Intelligence, 58, 161-205.

Mitra K., Gudib R.D., Patwardhanb S.E. and Sardara G. (2009). Towards resilient supply chains: Uncertainty analysis using fuzzy mathematical programming. Chemical Engineering Research and Design, 87, 967-981.

Montanari U. (1974). Networks of constraints: Fundamental properties and application to picture processing. Information Science, 7(2), 95-132.

Nadel B. (1989). Constraint Satisfaction Algorithms. Computational Intelligence, 5, 188-299. 
Richaud G. (2009). Outillage logiciel pour les problèmes dynamiques. Thèse de doctorat, Ecole nationale supérieure des techniques industrielles et des mines de Nantes, France.

Rota-Frantz K., Bel G. et Thierry C. (2001). Gestion des flux dans les chaînes logistiques. In Performance Industrielle et gestion des flux, Hermes Science, p. 153-187.

Sabin D. and Freuder E. (1994). Contradicting conventional wisdom in constraint satisfaction. Proceedings of the $11^{\text {th }}$ European Conference on Artificial Intelligence (ECAI'94), Amsterdam, The Netherlands, p. 125-129.

Sridharan S.V. et Laforge R.L. (2009). An analysis of alternative policies to achieve schedule stability. Journal of Manufacturing and Operations Management, 3, 53-73.

Stadtler H. and Kilger C. (Ed.) (2005). Supply chain management and advanced planning. $3^{\mathrm{e}}$ ed. Springer, Berlin.

Suwanruji P. and Enns S.T. (2006). Evaluating the effects of capacity constraints and demand patterns on supply chain replenishment strategies. International Journal of Production Research, 44, 4607-4629.

Tayur S., Ganeshan R. and Magazine M. (1999). Quantitative models for supply chain management. Kluwer Academic Publishers.

Verfaillie G. and Jussien N. (2005). Constraint solving in uncertain and dynamic environments: A survey. Constraints, 10(3), 253-281.

Vollmann T.E., Berry W.L. et Whybark D.C. (1997). Manufacturing planning and control systems. $4^{\text {th }}$ ed., McGraw-Hill Book Company, New York, NY.

Yano C.A. et Carlson R.C. (1987). Interaction between frequency of rescheduling and the role of safety stock in material requirements planning systems. International Journal of Production Research, 25, 221-232.

Zhang Y. and Yap R. (2001). Making AC-3 an optimal algorithm. Proceedings of the $17^{\text {th }}$ International Joint Conference on Artificial Intelligence (IJCAI'01), Seattle, WA, USA, p. 316-321. 